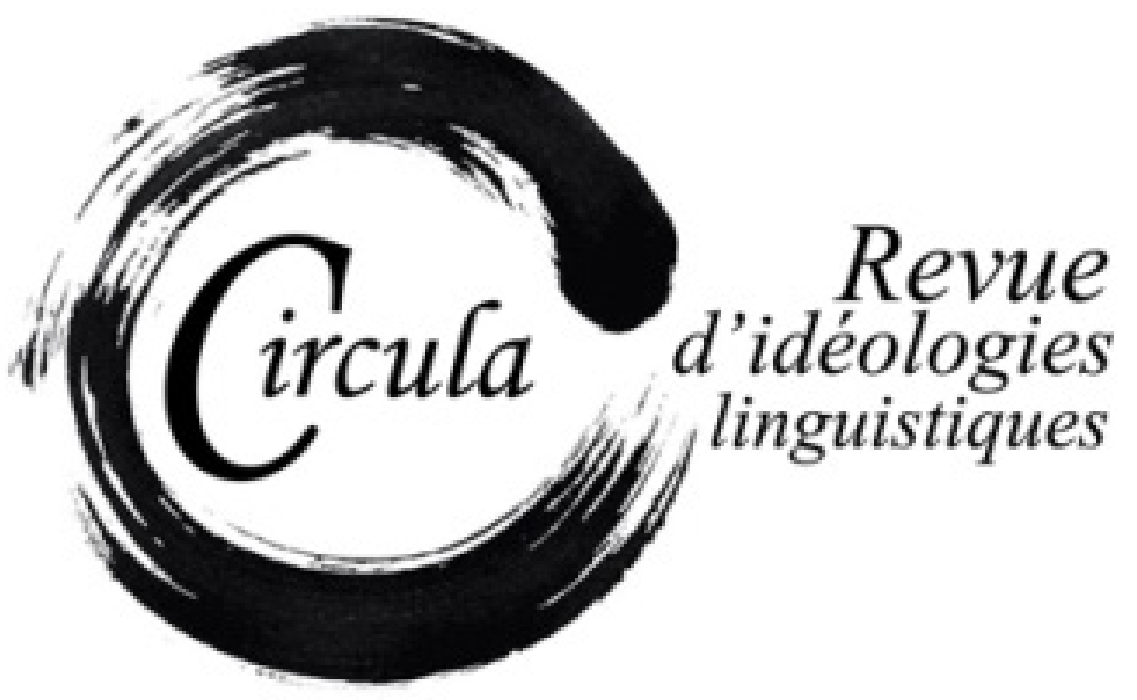

TITRE: INSÉCURITÉ LINGUISTIQUE CHEZ LES ENSEIGNANTS NON NATIFS DE FLE : LE CAS DES COLOMBIENS Auteur: Claudia Rincón Restrepo, École Jeannine Manuel- Paris, France

ReVue: Circula, NumÉro 12 : LA VULNÉRABILITÉ LINGUISTIQUE

DiRECTRICE: CLAUDIA TORRES CASTILLO

PAGES: $176-196$

ISSN: 2369-6761

URI: HTTP://HDL.HANDLE.NET/11143/18448

DOI: HTTPS://DOI.ORG/10.17118/11143/18448 


\title{
Insécurité linguistique chez les enseignants non natifs de FLE : le cas des Colombiens
}

\author{
Claudia Rincón Restrepo, École Jeannine Manuel- Paris, France \\ c.rinconrestrepo@ejm.net
}

\begin{abstract}
Résumé : Cet article s'intéresse à la notion d'insécurité linguistique dans les processus d'enseignement des langues étrangères, notamment celle ressentie par les enseignants de français non natifs en Colombie. Nous allons donc analyser les possibles raisons pour lesquelles les professeurs de français langue étrangère (FLE) colombiens ressentent cette insécurité, tout en abordant la notion et la représentation que se font ces enseignants du locuteur natif / non natif, ainsi que celles de langue maternelle ou première (LM/L1), qui en sont très proches. Nous présenterons également le statut et la place de la langue française en Colombie, avant d'évoquer le rôle joué par la formation des professeurs de FLE assurée dans les universités colombiennes. Enfin, nous présenterons quelques propositions pour tenter de remédier à ce sentiment d'insécurité, et améliorer ainsi non seulement la qualité de la formation et du travail des professeurs de FLE, mais aussi leur vie en général.
\end{abstract}

Mots clés : enseignement de FLE ; locuteur natif/non natif; insécurité linguistique

\begin{abstract}
This article examines the notion of linguistic insecurity in the process of learning/teaching foreign languages, in particular that felt by non-native French teachers in Colombia. We will therefore focus on the possible reasons why Colombian teachers of French as a foreign language (FLE) feel this insecurity, while presenting the notion and representation that these teachers make of the native / non-native speaker, as well as of the mother tongue or first langue (LM/L1), both being very close. We will also explore the status and place of the French language in Colombia, and finally discuss the role played by the training provided in Colombian universities for future language teachers. Finally, we will make some suggestions to address this feeling of insecurity and thus improve not only the quality of the training and the work of FLE teachers, but also their lives in general.
\end{abstract}

Keywords: teaching of FLE; native/non native speaker; linguistic insecurity 


\section{Introduction}

L'insécurité linguistique est un sujet largement discuté et étudié depuis l'apparition du terme dans les années 60. Pour le professeur de langue étrangère (LE) non natif, cette insécurité peut nuire à son développement professionnel, voire personnel. Elle peut avoir différentes origines, personnelles comme sociales, liées principalement aux représentations ou images que les enseignants et les professeurs des langues se font d'eux-mêmes et des autres, du processus cognitif et du modèle à suivre pour parvenir à «maitriser » une LE. Cette vulnérabilité linguistique parait s'accroitre en raison du sentiment d'illégitimité par rapport au professeur natif, ainsi qu'à l'idée de souffrir d'un déficit linguistique dû à un manque de pratique de la langue. Par ailleurs, le sentiment de ne pas suffisamment connaitre la culture de la langue cible, le fait d'avoir une prononciation incorrecte ou un accent très marqué et, enfin, le sentiment d'avoir reçu une formation médiocre peuvent également jouer un rôle important. Ayant identifié ces situations générant de l'insécurité chez les enseignants, comment y remédier?

\section{L'insécurité linguistique}

La notion d'insécurité linguistique apparait avec Labov en 1964. Haugen est pourtant le premier à utiliser ce terme, en $1962^{1}$, pour analyser l'insécurité dans des situations linguistiques où deux variétés d'une même langue coexistent. Il la décrit comme l'image négative ou le sentiment de dépréciation de soi-même ressenti par un locuteur exposé à une variété de sa propre langue plus valorisée que la sienne. Labov développe l'idée de Haugen (1962) et montre l'importance des facteurs extralinguistiques pour expliquer ce qui est linguistique. Ses études sur l'insécurité se centrent principalement sur la catégorisation sociale dans un même espace linguistique (l'anglais). D'après ses études, les pratiques les plus valorisées sont celles utilisées par l'élite (économique, politique, culturelle), qui s'imposent comme la norme officielle. Le prestige dont elles sont dotées n'a rien à voir avec leurs qualités intrinsèques, mais s'explique par celui dont sont investis les locuteurs de cette variété de langue comme norme sociale.

Plus tard, Francard, Lambert et Berdal-Masuy (1993: 13) théorisent cette notion à partir d'une étude sur des cas de diglossie en Belgique. Pour lui, l'insécurité linguistique est « la manifestation d'une quête non réussie de légitimité » et dépend de la prise de conscience du locuteur de la distance entre son idiolecte et une langue qu'il reconnait comme légitime, normalement celle apprise lors de la scolarisation. L'instruction donnerait en effet la connaissance de la norme sans pour autant assurer la maitrise pratique des registres, ce qui crée de l'insécurité.

1. Haugen parle de schizoglossie et de la norme linguistique. 


\subsection{L'insécurité linguistique en LE}

Calvet (1993) analyse les phénomènes relatifs à cette insécurité d'un point de vue inter-linguistique, c'est-à-dire en tenant compte de diverses situations de plurilinguisme. D’après lui, cette vulnérabilité apparait lorsque les locuteurs considèrent leur façon de parler, notamment dans les aspects phonétiques et syntaxiques, comme peu valorisante par rapport à un autre modèle considéré plus prestigieux qu'ils ne pratiquent pas. Il propose trois types d’insécurité linguistique:

- l'insécurité identitaire, qui apparait lorsque la langue ou variété pratiquée par le locuteur ne correspond pas à la communauté linguistique à laquelle il appartient ou à celle qu'il désire intégrer;

- l'insécurité formelle, liée au fait que le locuteur considère sa propre pratique linguistique comme non-conforme aux normes, ou à l'idée qu'il se fait de celles-ci ;

- l'insécurité statutaire, qui nait lorsque la langue ou variété pratiquée par le locuteur est perçue comme illégitime ou n'a aucun statut reconnu.

Selon Bourdieu dans son livre Ce que parler veut dire : l'économie des échanges linguistiques, la vulnérabilité linguistique chez les personnes s'exprimant dans une langue qui leur est étrangère surgit d'une non-maitrise des normes² ou de l'idée de ne pas les maitriser, et est liée à une volonté de parvenir à la forme considérée comme plus prestigieuse, ce qui peut amener à commettre des fautes et des erreurs.

Quand un locuteur se trouve dans une communauté linguistique où est pratiquée une langue qu'il ne maitrise pas, le sentiment d'insécurité linguistique se traduit chez lui par un effort conscient de correction afin de se rapprocher de l'usage jugé prestigieux. (Bourdieu, $1982: 104)$

Ainsi, les échanges entre un locuteur dit « natif ${ }^{3}$ » et un locuteur dit « non natif » peuvent entrainer des rapports de force qui, continuellement reproduits, constituent une forme de violence symbolique et qui, outre l'insécurité linguistique, peuvent mener à des représentations de soi extrêmement négatives.

2. Suivre la norme signifie respecter toutes les règles qu'une variété linguistique impose pour que l'usage soit correct pour celui qui veut l'imiter, l'utiliser ou la parler. Ces normes peuvent être perspectives, fictives, communicationnelles ou évaluatives.

3. Les ternes « natif » et « non natif » seront mis entre guillemets par la suite pour souligner le caractère relatif de ces notions. 


\subsection{Distinction entre locuteur «natif » et «non natif »: une source d'insécurité}

La figure du locuteur « natif » représente, de manière ambivalente, un fantasme et une source d'insécurité linguistique. Mais pourquoi le locuteur natif représente-t-il un idéal à suivre?

La notion de locuteur «natif » est introduite par la linguistique traditionnelle structurale. Celle-ci oppose de façon irréductible au sein de chaque langue le locuteur « natif » au locuteur « non natif ». Selon l'affirmation chomskyenne, l'objet de la linguistique serait ce natif, présenté comme un :

Ideal speaker-listener, in a completely homogeneous speech-community, who knows its language perfectly and is unaffected by such grammatically irrelevant conditions as memory limitations, distractions, shifts of attention and interest, and errors (random or characteristic) in applying his knowledge of the language in actual performance. (Chomsky, $1965: 3$, cité par Muni Toke, 2010 : 54)

De son coté, Love (2009 : 28), définit les locuteurs natifs comme «those who have been born and brought up to speak [a language] from birth, as their "mother tongue", in a family where the parents or other adults were brought up to speak the language from birth ».

Toutefois, pour Derivry-Plard, il s'agit de distinguer ce qui dans les notions de « natif » et « non natif » appartient à la catégorisation linguistique et à la construction sociale. En effet, la notion définie par Chomsky est, pour elle, une abstraction théorique où l'on voit la langue comme un système normatif et non comme une variation Dans son article Les enseignants «natifs » et «non-natifs » de langue(s): catégorisation linguistique ou construction sociale?, elle écrit:

Cette notion de «locuteur natif » a été particulièrement centrale pour l'élaboration de la grammaire générative développée par N. Chomsky. Il s'agissait de s'appuyer sur les intuitions grammaticales, lexicales et stylistiques d'un locuteur natif idéal, (c'est-à-dire aussi éduqué et «monolingue ») pour rendre compte du système de la langue décrit et de son fonctionnement comme capacité à générer un nombre illimité d'énoncés grammaticalement corrects ou acceptables à partir d'un nombre limité de signes. (...). La linguistique variationniste va s'opposer radicalement à cette vision «normative » de la langue, en remettant en question ces notions mêmes de «langue » et de «locuteur » comme définitions étanches et en les posant toutes deux selon des continua et un principe de diversité ; dans un même espace linguistique, il y a une diversité de langues (orales, écrites qui varient selon les âges, le sexe, l'origine sociale et géographique des divers locuteurs et selon les situations langagières et selon aussi que la compétence du locuteur diffère également de celle de l'auditeur). (Derivry-Plard, $2006: 101)$. 
Comme le dit cette auteure, la linguistique variationniste et plus encore la sociolinguistique, vont s'opposer à cette définition étanche et à cette vision structurale de la langue, remettant en question cette notion, et même celle de langue. Cette figure de locuteur natif sera aussi abondamment discutée en linguistique théorique, suivant ainsi le courant des études postcoloniales, dénonçant dans cette acception une position occidentalo-centralisée de la science linguistique, comme le montrent les travaux de Coulmas (1981) ou plus récemment de Davies (2003).

Dans son texte, Paikeday (1985) considère que cette notion de « natif » s'apparente davantage à un mythe propagé par les linguistes. D’autres chercheurs, comme par exemple Rampton (1990), proposent de remplacer ce terme par celui de locuteur expert, car ce dernier fait référence à l'expérience de locuteur dans une langue et non pas à son identité. Cook (2016), pour sa part, préfère le terme locuteur multi compétent, faisant référence aux niveaux de maitrise atteints dans différentes langues.

Le terme LM, étroitement lié à celui de locuteur natif, a également été remis en question, notamment par Urbain (1982: 8). Celui-ci considère que ce concept ne se laisse pas appréhender aisément et peut être défini de façons diverses. Pourrait-on alors lui donner un sens unique?

Du latin maternus, ce terme apparait pour la première fois au Moyen Âge en référence à la langue régionale parlée dans des contextes informels, en opposition à l'usage érudit du latin. Actuellement, il désigne la première langue apprise par un enfant à la maison de manière inconsciente, ce que l'on a souvent compris comme étant la langue de la mère. Par cette langue maternelle, l'enfant apprend la maitrise pratique des règles d'interaction sociale dans un ensemble culturel donné. Ces définitions ne sont cependant pas suffisantes pour couvrir toutes les réalités socio linguistiques existantes, car la langue de la mère n'est pas toujours la première langue apprise. Dans le cas de couples mixtes ou de milieux plurilingues, par exemple, elle peut être la langue véhiculaire ou commune entre les parents, la langue du père ou d'autres.

Pour tenir compte de ces ambiguïtés et confusions, la didactique des langues préfère l'usage de L1 (langue première) plutôt que maternelle. Davies (2003 : 16-17) observe ainsi qu'un enfant peut acquérir deux ou plusieurs langues simultanément. On peut ainsi dire qu'un enfant peut avoir deux ou plusieurs LM, mais il serait pourtant difficile d'établir laquelle est sa L1. D'autres chercheurs, comme Skutnabb-Kangas et McCarty (2018), définissent la LM selon quatre critères : l'ordre d'apprentissage (première langue apprise), l'identification (langue(s) avec la(es)quelle(s) le locuteur s'identifie ou est identifié), la compétence (la(es) langue(s) qu'il maitrise le mieux) et la fonction (la(es) langue(s) la(es) plus utilisée(s)). Ainsi, la ou les L1 d'un individu peuvent changer au cours de son existence, selon un principe évolutif et non statique.

Selon Dabène (1994) dans Torres-Castillo (2018 : 92-93), la langue maternelle est « celle dans laquelle s'est organisée la fonction langagière elle-même, en tant que fonction symbolique primordiale, et celle qui accompagne la construction de la personnalité ». D'après cette auteure, diverses considérations doivent être prises en compte pour définir la notion de LM, qui ne serait pas seulement 
la langue de la mère comme le dit l'étymologie. Elle peut être la première langue dans l'ordre d'acquisition, c'est-à-dire juste après la naissance, d'où les expressions fréquentes de « langue native » et de «locuteur natif ». La LM peut également désigner la langue la mieux connue par le locuteur, s'il en parle plusieurs, ou celle qu'il a apprise «naturellement ». Ceci signifie trois choses : on apprend cette langue sans s'en rendre compte, il n'existe pas d'intervention pédagogique quelconque et l'on l'apprend en parlant avec les autres.

\subsection{Distinction entre locuteur «natif » et professeur «natif »}

Derivry-Plard (2008 : 190) affirme que locuteur et professeur de langue ne doivent pas être confondus, car un locuteur est rarement enseignant de sa LM, et un professeur de LE est toujours un «natif » dans une ou plusieurs langues. Un «natif » peut en effet être très compétent dans l'usage d'une langue, mais ne pas avoir les compétences nécessaires pour l'enseigner. Le locuteur « natif » fait plutôt référence à une catégorisation linguistique quelque peu dépassée, alors que le terme « enseignant » fait référence à une catégorie sociale professionnelle dont les membres disposent tous, en droit, de la même compétence d'enseignement. Dans sa thèse de doctorat (2003), cette auteure expose les conclusions d'une analyse quantitative, comparative et multivariée comparant les résultats d'étudiants apprenant une LE avec un professeur « natif », et d'autres apprenant avec un «non natif ». Il apparait que les seconds ont obtenu de meilleurs résultats que les premiers, invalidant l'idée que l'enseignement d'un professeur «natif » est meilleur que celui d'un «non natif».

Malgré ces résultats gratifiants et encourageants, les professeurs « non natifs » se sentent toujours vulnérables, illégitimes. Derivry-Plard explique par exemple qu'en France les enseignants d'anglais non natifs ressentent de l'insécurité car les apprenants préfèrent avoir des professeurs natifs. Cela reflète une attitude générale et largement partagée dans l'opinion française. Dans les domaines économique, médiatique, politique et éducatif existe un consensus selon lequel un professeur natif serait plus compétent qu'un non natif.

D’après Coste (2008, cité par Torres-Castillo, 2017 : 142), le modèle sur lequel se basent les niveaux de compétence des enseignants en LE a été construit à partir de la figure du locuteur natif. C'est ce locuteur natif qui sait prononcer correctement les mots, a une vaste connaissance lexicale et connait très bien sa culture. Dans l'imaginaire des enseignants et des apprenants, ce «natif » est le locuteur idéal et légitime et parle une langue standard. À ce propos, Muni Toke nous dit : 
L'idéalisation chomskyenne, en faisant de la variation linguistique une quantité négligeable dont le scientifique devrait se défaire, aurait contribué à renforcer la centralité du locuteur natif et de la langue standard dans les sciences du langage. Or, ces deux notions sont des idéologies politiques, associées à la discrimination ethnique et à l'homogénéisation forcée des pratiques linguistiques; pourtant, la définition chomskyenne est apolitique, décontextualisée, sans aucune dimension sociale et sociologique. (...) On pourra reprocher à cet enchainement apparemment logique la confusion de deux ordres : celui du natif, description commune à valeur sociale et politique, et celui de l'idéal, modélisation scientifique explicitement « réductionniste » et « extractionniste». (Muni Toke, 2013:8)

Cet amalgame entre locuteur « natif » et « idéal » relève donc d'une confusion, d'une construction sociale et non d'une réalité. Il faudrait, comme le disent Candelier et Castellotti (2013), disqualifier cette notion dans le domaine de l'enseignement des langues, car il n'existe pas un seul modèle de locuteur natif. Selon Muni Toke:

Ce terme «natif » est également porteur d'une valeur ethnico raciale implicite : l'authenticité et la légitimité du locuteur natif tiennent à l'appartenance culturelle qu'on lui prête - voire à son phénotype comme dans l'exemple ci-dessus. On voit quels raccourcis il a soudainement fallu accepter, pour que la « compétence » supposée d'un locuteur se retrouve corrélée à son origine ethnique. Et pourtant, la construction d'inégalités sociales à partir de ce présupposé est monnaie courante, et il y aurait bien ainsi des natifs qui seraient plus natifs que d'autres. (Muni Toke, 2003 : 6)

\section{L'insécurité chez l'enseignant de FLE « non natif »}

Tant dans son entourage que dans son milieu professionnel, l'enseignant de LE est considéré comme un modèle, compétent ou capable non seulement d'enseigner, mais aussi de communiquer sans problème, de répondre, de poser des questions et de discuter en utilisant la langue étrangère dans laquelle il a suivi une bonne formation et a obtenu un diplôme universitaire. L'image que son entourage a de ce professeur expert influe sur sa sécurité. Il est difficile pour un enseignant, voire honteux, de confesser qu'il rencontre des difficultés dues à l'insécurité linguistique, qui, comme dit plus haut, peuvent avoir des origines diverses. Nous évoquerons ici celles relatives à l'insécurité identitaire évoquée par Calvet (1993), liée à la conscience de ne pas être « natif » et aux images que les enseignants/apprenants se font de ce professeur « natif », et à l'insécurité formelle, qui peut provenir du sentiment de ne pas maitriser les normes en raison d'un manque de pratique ou d'une formation insuffisante.

Concernant l'insécurité linguistique chez les enseignants « non natifs » de LE, Roussi (2009 : 21) écrit, dans sa thèse de doctorat, que leur conscience normative serait partagée entre la langue qu'ils 
ont apprise, qu'ils enseignent avec les fautes, les imperfections ou tout simplement les particularités des locuteurs « non natifs » et la perception stéréotypée d’un locuteur « natif » supposé idéal.

Dans la tradition de l'enseignement des LE et dans l'opinion publique, la distinction, voire hiérarchisation, entre locuteur « natif » et « non natif » a toujours été présente. On peut même parler d'une opposition entre ces deux concepts, voire d'une confrontation (Planet, 2014). Cette idée s'est renforcée depuis l'apogée de la méthode directe à la fin du XIX` siècle et au début du XX siècle, où l'exercice privilégié était la conversation en LE sans devoir passer par la langue maternelle et où le professeur ne devait pas donner d'explication sur le fonctionnement de la langue. Durant cette période, n'importe quel locuteur natif pouvait en effet devenir professeur de LE, l'objectif étant simplement de parler et d'apparaitre aux apprenants comme un modèle à suivre.

Bien que cette méthode ne soit plus en vigueur, la figure du professeur de LE « natif » reste, dans divers contextes, insubstituable et idéale : il connait sa propre culture et maitrise sa LM, prononce correctement les mots, est authentique, légitime et fiable.

Roussi (2009) aborde l'insécurité linguistique vécue par les professeurs de FLE en Grèce comme un sentiment de déficit linguistique, de peur et de honte de commettre des erreurs pendant leurs cours, ou de reconnaitre qu'ils manquent de connaissances linguistiques et culturelles. Ils ressentent cette insécurité non seulement dans le cadre académique, mais aussi en dehors de la salle de classe, par exemple dans les échanges de la vie quotidienne avec des natifs ou des collègues. Dans la salle de classe, le professeur développe des stratégies d'évitement qui peuvent l'aider à contourner les difficultés. En revanche, dans la vie réelle, quand la communication n'est pas guidée, le fait de mal prononcer un mot, de l'oublier ou de l'utiliser de manière incorrecte, peut être ressenti comme une mise en défaut de sa maitrise de la langue, et de ses compétences en général. Cette vulnérabilité peut donc affecter non seulement sa vie professionnelle, mais aussi sa vie privée.

L'exemple des professeurs grecs s'apparente, là aussi, à l'expérience des enseignants en Colombie, qui peut devenir tellement stressante que certains professeurs partent en France ou dans un pays francophone, en quête de légitimité4. Ils veulent poursuivre leurs études et ainsi revendiquer leur compétence en tant que professeurs de FLE, avec l'espoir de revenir plus crédibles au pays. D'autres, une fois installés dans un pays francophone, se tournent plutôt vers l'enseignement de leur propre L1 - l'espagnol - dans lequel ils se sentent plus reconnus et légitimes.

Pour Roussi, le manque de pratique, en dehors du contexte scolaire, de la langue enseignée peut être une autre source d'insécurité pour les professeurs. La situation est similaire en Colombie, en raison du statut du français dans le pays. La possibilité de parler cette langue en dehors d'un contexte académique est en pratique très faible. La situation s'est toutefois améliorée ces dernières années grâce aux programmes d'échanges académiques entre la France et la Colombie, l'arrivée massive

4. Ainsi, 56\% des 3000 Colombiens étudiant en France sont dans des filières linguistiques. 
des nouvelles technologies et l'ouverture du pays au tourisme international, après avoir été isolé par plus de 50 ans de conflit armé.

\subsection{L'insécurité linguistique chez les enseignants « non natifs » de FLE en Colombie}

En Colombie, l'opposition entre « natif » et « non natif » perdure, principalement dans le milieu académique (Rincón Restrepo, 2016 :327), créant ainsi une différence de statut entre ces deux profils, ainsi qu'un sentiment d’illégitimité et de vulnérabilité chez les enseignants « non natifs » de FLE. Ceux-ci éprouvent alors des difficultés à se positionner vis-à-vis du professeur francophone natif. Ils ont le sentiment, de par leur perception propre et celle de leur entourage, que leur compétence sera toujours dévalorisée face à l'image parfaite du «natif ».

Dans l'enseignement des LE en Colombie, la progression des étudiants et aussi la compétence du personnel enseignant sont mesurées selon l'image du locuteur « natif » idéal. Ce qui a des répercutions parfois très négatives sur leur motivation, car les apprenants, en constatant qu'ils n'atteindront jamais le niveau d'un natif, peuvent se décourager. Comme le dit Torres-Castillo:

Les idées stéréotypées des langues et des locuteurs empêchent de comprendre l'enseignement-apprentissage des langues comme une relation des différentes connaissances linguistiques, et elles peuvent à la fois devenir de vrais empêchements pour la maitrise de la langue et la cause de diverses déceptions. (Torres-Castillo, 2018: 53)

Cette idéalisation du natif se voit parfois reflétée dans les offres d'emploi, car les institutions cherchent des «natifs » pour plus tard mieux vendre leurs services, renforçant ainsi l'idée stéréotypée du « natif » auprès de la population : lui seul peut enseigner sa culture ou comment bien prononcer sa langue, et il lui suffit pour cela d'être en contact avec ses étudiants, ce qui n'est pas réaliste. Cette situation, souvent vécue dans des pays comme la Colombie, où la plupart des professeurs de FLE sont « non natifs » (Colombiens) ${ }^{5}$, génère un énorme désavantage entre les professeurs formés dans les universités colombiennes ayant acquis des connaissances linguistiques et pédagogiques solides, et les natifs qui n'ont parfois même pas de formation en enseignement de leur LM. Le désavantage est, bien sûr, encore plus marqué si ceux-ci ont suivi une formation pédagogique et didactique en LE. Avoir un professeur « natif » est devenu un privilège dans ce pays sud-américain. Ils sont généralement mieux payés, même quand ils ont une formation plus médiocre. Dans cette échelle professionnelle, l'enseignant latino-américain subira, outre l'insécurité linguistique, une discrimination à l'emploi.

\footnotetext{
5. Si, d'après le ministère de l'Éducation nationale en Colombie, la majorité des professeurs de LE sont des Colombiens, la proportion de professeurs natifs varie selon le type d'établissement. Les écoles bilingues internationales recrutent principalement des directeurs et des enseignants d'origine étrangère, et offrent notoirement de meilleures rémunérations que les autres. Dans les écoles bilingues nationales, les enseignants sont tous bilingues mais principalement d'origine colombienne. Dans les écoles non bilingues, seuls les professeurs de LE sont bilingues, et en quasi-totalité des Colombiens formés dans les universités du pays. Information disponible en ligne sur www.mineducacion.gov.co/1759/ w3-article-364450.html?_noredirect=1.
} 
Une autre des principales sources d'insécurité linguistique chez les professeurs colombiens de FLE est le fait d'avoir un accent étranger, non natif, ce qui est souvent perçu, selon Cook (2014, cité par Torres-Castillo, 2017 : 142) comme un échec dans l'apprentissage de la LE. En effet, nous savons qu'acquérir un accent natif, bien que possible, est très difficile, principalement pour des personnes commençant à apprendre une LE à l'âge adulte, cas de la plupart des étudiants des licences de formation de futurs professeurs des langues en Colombie (au moins pour ce qui concerne le français).

\begin{abstract}
(...) un individu qui n'a pas appris une langue dans l'enfance et ne l'apprend qu'à l'âge adulte la parle rarement sans accent. La raison en est que son appareil phonatoire n'aura pas assimilé les mouvements qui permettent de produire correctement les sons d'une langue donnée. La production de sons de la langue maternelle s'inscrit dans des algorithmes moteurs mémorisés par l'appareil neuromusculaire. Elle privilégie certains muscles et en laisse d'autres en jachère. Ensuite cette mémoire du corps contrarie l'émission normale de la plupart de sons des autres langues. Ainsi est de beaucoup d'éléments de culture qui sont autant de répertoires d'action et de pensées permanents qui nous façonnent parce que nous les avons incorporés. (Warnier, 2004 : 10, cité par Torres-Castillo, 2017 : 143)
\end{abstract}

Mais le fait d'avoir un accent n'a rien à voir avec la qualification pédagogique, voire avec la compétence linguistique. Cette idée réduisant la capacité d'enseigner une langue à la question phonologique reproduit, d'une certaine façon, la dichotomie entre dominant (le natif) et dominé (le non natif), comme le dit Torres-Castillo (2018). Jusqu'à aujourd'hui, les professeurs de LE sont souvent classifiés par rapport à leur L1 et non par leurs capacités linguistiques et pédagogiques.

Avoir un accent n'est pas le seul complexe des professeurs colombiens de LE. Toute marque de L1 dans leurs discours peut être mal perçue. Pourtant d’après Galisson (1986 : 52), la LM est présente de manière visible ou invisible dans l'apprentissage d'une LE, est en fait la première référence, le fil conducteur, une piste universelle. Les études psycholinguistiques montrent que la L1 est indéniablement omniprésente dans le cerveau des apprenants d'une LE/L2. En fait, faire appel à sa LM lors de l'apprentissage d'une LE est une pratique naturelle conforme à toute situation de communication de contact des langues (Causa, 2002, cité par Benamar, 2014 : 139). Ainsi, la LM ne devrait pas être vue comme un obstacle ou exclue de l'acte pédagogique en LE, mais plutôt mise à profit dans le processus d'apprentissage en tant qu'outil.

Par ailleurs, dans l'imaginaire de beaucoup de Colombiens, les natifs de langue française ne sont pas seulement supérieurs concernant la prononciation correcte et l'enseignement du français, mais dans tous les domaines. Il existe en effet en Colombie - et dans la plupart des territoires jadis colonisés - un sentiment d'infériorité par rapport aux européens, pour des raisons historiques, comme le dit Torres Castillo dans sa thèse (2018) à propos de la situation des enseignants non natif de FLE au Mexique. Lors de la colonisation de ce pays, par exemple, les relations qui s'établirent entre les Espagnols et les Indigènes étaient des relations de pouvoir déséquilibrées. Les Espagnols, convaincus de leur supériorité raciale et culturelle, considéraient les Indigènes comme des êtres peu évolués. 
De leur côté, les Indigènes les considéraient aussi comme des êtres supérieurs qu'ils respectaient et reconnaissaient comme tels. L'idéalisation de l'étranger au Mexique, et dans tous les territoires colonisés par les occidentaux, n'est peut-être que le résultat de cette vieille coutume qui préparait les individus à respecter les dieux. Torres-Castillo nous dit:

Cet imaginaire s'est construit à partir des récits de la Conquête et à partir aussi de quelques documents indigènes comme les codex. Malheureusement l'histoire a été plutôt écrite la plupart du temps par ceux qui ont vaincu aux yeux du monde, nous laissant juste une version occidentale de cet évènement historique. (Torres-Castillo, 2018: 155)

Cette idéalisation de l'étranger est donc très ancrée dans les mentalités latino-américaines. Comme nous le savons, la société coloniale en Amérique latine était organisée par castas. Celle qui se trouvait en haut de l'échelle était celle des Espagnols et des criollos (fils des Espagnols nés en Amérique). Celles des natifs et des noirs se trouvaient tout en bas. Ainsi, par exemple, quand un individu de l'époque voulait poursuivre ses études universitaires pour se préparer à accéder à des postes administratifs, il devait d'abord démontrer qu'il était un honorabilis vir, purus ab omni macula sanguinis atque legitimis et natalibus descendens, c'est-à-dire un « homme honorable, pur de toute tache de sang et descendant de parents légitimes ». Cette «tache » de sang fait référence au métissage avec les noirs ou les indiens. Les groupes de privilégiés - les Blancs européens - craignaient en effet l'avancée progressive des droits du peuple et du pouvoir politique et économique que commençait à obtenir la population métisse. Ces préjugés d'impureté étaient très présents et persistent, incroyablement, même aujourd'hui et l'Européen est encore vu comme supérieur (Rincón Restrepo, 2016 : 25, 27). Plus tard, au cours du XVIIle siècle, apparait en Colombie une certaine idéalisation de la France et des Français. À cette époque, le français est la langue de l'élite, du monde civilisé. De fait, à cette époque, la société hispano-américaine, comme le monde entier, est divisée entre civilisés et barbares. Cette dichotomie rend possible et légitime le colonialisme européen, notamment en Afrique et en Asie. Les Européens, se considérant supérieurs aux autres peuples, entreprennent de civiliser les peuples natifs de ces continents. Ce qui n'est plus possible aux Amériques, d'abord en raison d'une volonté d'indépendance dans presque tous les territoires, acquise récemment avec beaucoup d'efforts, ensuite parce que les États-Unis, dirigés par le président James Monroe, établissent la doctrine Monroe ${ }^{6}$ en 1823, considérant toute nouvelle intrusion des États européens comme un acte d'agression requérant l'intervention des États-Unis, et enfin, car, en Amérique latine, l'élite, d'origine espagnole, cherche à civiliser ou à « européaniser » la population de la nouvelle nation, rejoignant ainsi l'objectif des Européens. Ainsi, cette dichotomie, rendue publique en Argentine et ultérieurement dans toute l'Amérique hispanique par Domingo Faustino Sarmiento dans son livre Facundo, est à l'origine de la bataille pour l'instruction publique qui se développe en Amérique latine au XIX'e siècle. L'élite criolla, descendant des Espagnols, voit la société européenne comme une société modèle, civilisée, tandis que celle des nouvelles nations américaines est vue comme barbare et inférieure. Elles doivent

6. La doctrine Monroe : elle a rendu fameuse la phrase L’Amérique pour les Américains, et détermine la politique extérieure des États-Unis. 
être civilisées coûte que coûte, grâce à l'éducation, et à travers une langue européenne. L'espagnol, étant la langue maternelle de l'élite et la plus répandue - mais pas forcément la plus parlée - sur le territoire colombien, est donc choisie comme langue nationale. Toutefois, les idées véhiculées en langue espagnole sont, pendant cette période, celles des Européens. L'élite criolla continue alors à regarder vers la France et l'Angleterre, idéal de culture et de progrès, summum de la civilisation (Rincón Restrepo, 2016: 50). La francophilie est donc très présente non seulement en Colombie mais aussi dans toute l'Amérique latine au XVIII siècle. La France a marqué l'histoire du pays et a été, jusque récemment, le modèle à suivre en matière éducative comme dans bien d'autres domaines. Au XIXe siècle, le gouvernement de Simón Bolívar, très influencé par les idées républicaines de la France, se tourne principalement vers ce pays en matière d'éducation et tente de copier ses modèles éducatifs. Le choix d'enseigner le français est, lui, le fruit de l'influence de la culture française depuis le XVIIe siècle. Le rôle de la langue française est, surtout, de cultiver et civiliser les jeunes gens de la nouvelle république. Pendant le XIXe siècle, la langue française reste très importante pour des raisons historiques, culturelles, et diplomatiques. Les idéaux républicains français se répandent d'abord dans plusieurs domaines, notamment juridique et éducatif, grâce aux élites libérales, qui écrivent et parlent couramment le français. Les programmes éducatifs et les lois instaurés suivent ainsi le modèle français et s'inspirent des idées du siècle des Lumières. Jusqu'à la première moitié du XX siècle le français est considéré comme langue de civilisation, et est la première langue étrangère dans le pays. Les Français, quant à eux, sont vus comme des gens cultivés, par opposition aux Colombiens, mettant en évidence la dichotomie civilisation/barbarie discutée plus haut. Nous constatons ainsi que la rencontre avec l'étranger, si elle peut permettre de mieux apprécier sa propre communauté, sa propre société, sa propre « culture », peut être également source de frustration personnelle, comme c'est souvent le cas des Colombiens face aux Français, et en particulier pour les professeurs de FLE non natifs.

\subsection{La figure du locuteur natif dans l'enseignement des langues en Colombie}

Dans le contexte de l'apprentissage du FLE en Colombie, le locuteur « natif » idéal reste le Français de la métropole. En effet, le français académique est perçu comme celui parlé en France, les autres expressions de la francophonie n'étant pas considérées comme légitimes (Rincón Restrepo, 2016 : 327). Le statut de la langue française varie selon qu'elle est langue maternelle, officielle, d'usage, de culture et si elle est parlée par une majorité ou une minorité de locuteurs, selon Lise Gauvin (2008: 28). Les « francophones » des zones où elle n'est pas langue maternelle ou est une langue minoritaire sont vus comme des usagers de la langue de second plan. Jusqu'à la première moitié du XXe siècle, on reconnaissait seulement une langue française, celle de la France, standardisée par le français parisien bourgeois de l'époque de la Révolution française. Ainsi, le français des autres régions du globe était considéré comme dialecte : celui du Canada était vu comme un mauvais mélange entre anglais et français, par exemple, et le français des anciennes colonies est souvent vu comme une imitation du véritable français. Par ailleurs, la France accepte encore mal que les identités nationales ne soient pas univoques, comme le dit Charles Bonn (2008 : 45), peut-être à cause d'une tradition jacobine 
postulant l'unité de la langue. Si les francophones de la « périphérie » ne sont pas reconnus comme des véritables «natifs » en Colombie, la situation est encore pire pour les enseignants « non natifs ». Ils sont souvent soupçonnés de ne pas maitriser « parfaitement » la langue qu'ils enseignent, de ne pas avoir le niveau d'un Français et d'être, tout simplement, de mauvais professeurs.

Si le français hexagonal est perçu en Colombie comme " authentique ", c'est parce que le contact avec la francophonie, au moins jusqu'au début du XXI siècle, y a souvent été assuré en exclusivité par des acteurs hexagonaux (Rincón Restrepo, 2016 : 328). L’Amérique latine en général est en effet l'une des régions du monde où la prégnance de la culture française est la plus forte, comme le fait remarquer Silva (2011 : 6). Ainsi, il est compréhensible que le français de France soit évoqué comme modèle dans l'enseignement du FLE, où l'on cherche à faire apprendre le français hexagonal selon une normativité linguistique pédagogique, en argumentant qu'elle permettrait de structurer plus facilement le savoir.

\subsection{Le statut du français en Colombie et la formation des professeurs de FLE: une autre source d'insécurité}

En Colombie, le français a actuellement le statut de deuxième langue étrangère, mais occupa longtemps une première place incontestable. C'est dans la période de l'après-guerre que le français commence véritablement à perdre de son influence face à l'anglais, lorsque la mise en place de lois éducatives se conforme à des pressions géopolitiques. Le français, dont l'enseignement devient peu rentable, disparait ainsi progressivement du secondaire, laissant l'anglais comme seule langue étrangère obligatoire (Rincón-Restrepo, 2020 : 50). La propagation de cette langue et de la culture américaine accompagne l'accroissement du commerce international, le développement des moyens d'information et les avancées scientifiques et techniques, qui engendrent des bouleversements économiques, sociaux et culturels, ainsi que des changements dans les mentalités.

Dans les années 1990, le gouvernement colombien reconnait dans la Nouvelle Constitution de 1991 le caractère multilingue et pluriculturel du pays. Malgré cela, il promeut essentiellement un bilinguisme espagnol-anglais dans le secteur éducatif, comme l'illustre le Programme national de bilinguisme 2004-2019 (PNB). La diversité linguistique est donc encore entendue comme une simple dualité entre la langue officielle et une seule langue étrangère. Le manque d'harmonisation des politiques linguistiques dans le domaine éducatif conduit à l'absence de langues étrangères autres que l'anglais dans les programmes de formation des enseignants des langues, et à une représentation très réduite des langues nationales autres que l'espagnol' .

7. En Colombie, $98 \%$ de la population parle espagnol, le reste parlant des langues nationales ou des langues créoles. 
Les tensions politiques se manifestent alors dans les universités colombiennes qui décident de réformer, voire de supprimer, les formations de professeurs de FLE. Le français perd complètement sa place dans l'enseignement secondaire vers la fin du XX e siècle et lutte pour garder un espace dans la formation universitaire des professeurs de cette langue.

Au début du XXI e siècle, les entités en charge de la validation de cette formation de professeurs de LE décident alors d'annuler les épreuves de langues autres que l'anglais de l'examen d'État (pruebas Saber Pro) à la fin des licences de formation universitaire ${ }^{8}$. La compétence d'un professeur de LE ne s'évalue ainsi qu'en anglais (en français si la formation est spécifique dans cette langue9) ce qui nuit à la qualité de la formation et qui peut, à long terme, engendrer une insécurité linguistique chez les professeurs, qui se sentent mal préparés à enseigner la langue française, perçue en Colombie comme une langue difficile et sophistiquée nécessitant de longues études pour être maitrisée.

Actuellement, les universités colombiennes, désireuses d’apparaitre en bonne place dans les classements internationaux, concentrent leurs programmes de formation des professeurs de LE sur la formation en anglais et délaissent celle dans d'autres LE, par exemple en accordant moins d'heures aux cours de littérature et culture françaises, ou en accordant peu de budget au recrutement de professeurs de FLE qualifiés. Ainsi, en 2015, le ministère de l'Éducation, après analyse des résultats des examens Saber Pro des années précédentes, a décidé de supprimer la formation en français dans des licences octroyant un double ou triple titre par diplôme, car les étudiants y obtenaient des résultats plus médiocres en anglais que ceux des licences octroyant un seul titre en cette langue. Cette décision n'a toutefois pas encore été appliquée, face à l'opposition d'une partie du corps enseignant, car elle pourrait entrainer la disparation presque totale du français dans le cadre académique universitaire, qui ne serait alors enseigné au niveau licence que dans deux universités du pays.

Pour l'instant, les victimes de ces politiques éducatives sont les étudiants de ces licences de formation de professeurs de LE, qui devenus enseignants ressentiraient de l'insécurité à l'idée d'avoir reçu une formation médiocre en langue et culture françaises, ce qui est, de fait, le cas. Dans une étude réalisée en 2014 en Colombie (Rincón-Restrepo, 2016), les étudiants dans des licences de formation de futurs professeurs de FLE octroyant un double titre anglais/français, estiment avoir reçu une meilleure formation en anglais qu'en français.

Néanmoins, ces dix dernières années, les entités chargées de la diffusion de la langue française ont commencé à « lutter » avec plus de force contre cette marginalisation du français dans les politiques linguistiques colombiennes. De nouveaux accords entre les institutions éducatives permettent une

8. Examen que doivent passer tous les étudiants à la fin de leur licence (BAC +5 en Colombie). Dans le cas des licences de formation de professeur de LE, l'examen Saber PRO évalue la connaissance pédagogique, la connaissance en didactique des LE, la connaissance linguistique en anglais et la connaissance de la problématique éducative en Colombie des futurs enseignants.

9. Parmi les 32 universités publiques et 49 privées du pays, 16 offrent une licence en formation initiale pour les professeurs de français. Parmi ces 16 programmes, deux proposent une formation exclusivement en français. 
mobilité des étudiants colombiens vers la France et le Canada, et Campus France, une agence gouvernementale française travaillant sur la promotion de l'éducation supérieure et la mobilité internationale née en 2006, est chargée de gérer toutes les démarches des étudiants désirant poursuivre leurs études en France pour compléter leur formation. Ces efforts ont permis, ces dernières années, un nouvel essor de l'enseignement du français en Colombie, principalement dans le secteur de l'enseignement privé dans des instituts des langues. Ils reflètent, en grande partie, une prise de conscience des pays européens, qui œuvrent avec une énergie nouvelle à diffuser leurs langues dans le monde. Le français, en tant que deuxième langue étrangère la plus apprise en Colombie et seule capable de faire contrepoids à l'anglais, joue un rôle moteur dans cette promotion de la diversité linguistique.

Par ailleurs, l'enseignement obligatoire du français dans les collèges et lycées en Colombie va être réintroduit. En 2010, le Mémorandum d'entente pour l'enseignement du français dans les institutions éducatives publiques en Colombie a été signé entre l'Ambassade de France en Colombie, l'Alliance colombo-française et le ministère de l'Éducation nationale. Ce mémorandum est le résultat d'un projet conduit depuis 2008 par un groupe d'universités publiques et par l'Association de professeurs de français (ACOLPROF), l'Alliance colombo-française et l'Ambassade de France, qui, après plusieurs années d'absence de cette langue dans les institutions publiques du pays, souhaitent que cet enseignement réintègre les programmes de formation (González, 2010). Le ministre français de l'Éducation nationale, Jean-Michel Blanquer, a également exprimé cette volonté lors de sa visite en Colombie en septembre 2019.

Dans ce mémorandum, toutes les parties considèrent la création des programmes éducatifs linguistiques et culturels indispensables au développement et à l'apprentissage de langues autres que l'espagnol. Pour sa part, l'Ambassade française en Colombie s'engage à élaborer et à mettre en place un programme suivant les orientations pédagogiques devant être établies avec le ministère et l'Alliance française; à coordonner la participation des organismes français dont l'alliance est considérée pertinente pour la mise en marche du programme; à contribuer à l'organisation, la préparation et la convocation d'événements académiques favorisant la réflexion et l'échange d'expériences en relation avec le programme. Ainsi, le français a commencé à être réintroduit dans l'éducation secondaire depuis 2012 dans quelques institutions, qui servent donc d'étude pilote.

\section{Conclusions}

L'insécurité linguistique chez les enseignants non natifs de FLE en Colombie provient de sources diverses, et rejoint celle ressentie par d'autres professeurs de LE non natifs dans le monde, comme le démontrent diverses études. D’après les catégories proposées par Calvet, ils ressentent principalement une insécurité identitaire, liée aux préjugés et aux représentations que se font les enseignants de langues du professeur « natif » et de ce qu'est un francophone, et une insécurité formelle, car ils considèrent que leur pratique linguistique est souvent non conforme aux normes. Cela est notamment lié à l'idée d'avoir reçu une formation moins bonne en français qu'en anglais dans les licences 
de formation de futurs de professeurs de FLE des universités colombiennes. Ces licences sont sujettes aux politiques linguistiques et éducatives établies au détriment des licences autres que celles liées à l'enseignement de l'anglais. S'y ajoute également la difficulté de mettre en pratique, dans la vie courante, leurs connaissances en LE.

Pour réduire le sentiment de vulnérabilité issu de l'insécurité identitaire, il serait bénéfique de revoir la formation des futurs professeurs de FLE dans ces programmes universitaires et de remplacer le paradigme actuel par une didactique du plurilinguisme. Il convient donc de dépasser la dualité «natif »/« non natif » et de redéfinir les objectifs de l'enseignement d'une LE, encore très centré sur un cadre "monolingue », afin de mettre les enseignants « non natifs » davantage en confiance. Aujourd'hui, l'objectif de l'apprentissage d'une langue n'est plus d'imiter un locuteur modèle ou idéal, mais d’apprendre peu à peu la langue et la culture qu'elle véhicule. Ainsi :

La pleine prise en compte du caractère profondément «pluri » de l'apprentissage des langues nécessite une rupture radicale avec l'enseignement traditionnel de type « monolingue/monoculturel » des langues et avec la formation tout aussi « monolingue/monoculturelle » des enseignants de langue(s). (Derivry-Plard, $2006: 107)$

Il faut par ailleurs accepter que, comme le démontrent plusieurs études, un natif quelconque ne peut remplacer un professeur de langue formé pour enseigner une LE (Cuq, 2003 : Torres-Castillo, 2018 : 247), mais devrait plutôt être considéré comme un « informateur » et comme « une ressource d'apprentissage ». L'enseignant doit alors guider, donner des pistes et fournir des stratégies d'apprentissage aux étudiants pour qu'ils deviennent compétents en LE, ce qui ne requiert pas d'être natif, mais d'obtenir de solides compétences d'enseignement. D'où la nécessité de formations de qualité. L'idée de former des individus avec une « maitrise parfaite » de la langue cible doit être oubliée, au profit d'une vision plus dynamique de la langue et de son processus d'apprentissage. Cette logique traditionnelle nous empêche de voir, comme le dit Torres-Castillo (2018: 383) que l'apprenant de LE, ici le professeur de LE, est à la fois un «natif » et un «non natif », et donc un locuteur plurilingue.

Quant à l'insécurité formelle, il est nécessaire de soutenir les licences de formation pour les futurs professeurs de LE dans les universités, notamment celles octroyant un double titre - professeur d'anglais et de français langue étrangère -, en menant une réflexion sur les transversalités didactiques entre les deux langues, car si ces programmes disparaissent, comment seront formés les futurs professeurs de FLE requis lorsque le français sera réintroduit dans le secondaire ? La création de programmes uniquement consacrés à la formation de professeurs de français n'est pas économiquement viable dans l'immédiat. À Bogotá, une formation de ce type peut attirer un nombre suffisant d'étudiants, puisqu'il existe un marché relativement vaste pour les diplômés. Ce pourrait être le cas aussi à Medellín et à Cali, mais pas dans le reste du pays.

Enfin, une nouvelle légitimité doit également être reconnue à l'enseignement de langues autres que l'anglais dans les secteurs du secondaire et universitaire, en accord avec les préconisations du 
CECR (Cadre européen commun de référence) selon lesquelles un apprenant ne doit pas uniquement maitriser une seule langue cible, mais être compétent dans plusieurs langues à différents degrés. 


\section{Références}

Benamar, Rabéa (2014), « La langue maternelle, une stratégie pour enseigner/apprendre la langue étrangère », Multilinguales, n³, p. 139-158.

Bonn, Charles (2008), «Pour un comparatisme français ouvert à la francophonie et aux métissages culturels : plaidoyer en forme de polémique », dans Ingse Skattum et Karin Holter (dir.), La francophonie aujourd'hui : réflexions critiques, Paris, L'Harmattan, p. 41-48.

Bourdieu, Pierre (1982), Ce que parler veut dire : l'économie des échanges linguistiques, Paris, Fayard.

Calvet, Louis-Jean (1993), La sociolinguistique, Paris, Presses universitaires de France.

Candelier, Michel et Véronique Castellotti (2013), "Didactique(s) du(des) plurilinguisme(s) », dans Jacky Simonin et Sylvie Wharton (dir.), Sociolinguistique du contact : dictionnaire des termes et concepts, Lyon, ENS Éditions, p. 179-221.

Causa, Maria (2002), L'alternance codique dans l'enseignement d'une langue étrangère : stratégies d'enseignement bilingues et transmissions de savoirs en langue étrangère, Bruxelles, Peter Lang.

Cook, Vivian (2016), « Where Is the Native Speaker Now ? », TESOL Quarterly, vol. 50, nº 1, p. 186-189.

Coulmas, Florian (1981), A Festschrift for native speaker, La Haye, De Gruyter Mouton.

Cuq, Jean-Pierre (dir.) (2003), Dictionnaire de didactique du français langue étrangère et seconde, Paris, CLE International.

Davies, Alan (2003), The native speaker: Myth and reality, Clevedon, Multilingual Matters.

Derivry-Plard, Martine (2003), Les enseignants d'anglais "natifs » et " non-natifs » : concurrence ou complémentarité de deux légitimités, thèse de doctorat, Université Paris 3/INALCO.

Derivry-Plard, Martine (2006), "Les enseignants "natifs" et "non-natifs" de langue(s) : catégorisation linguistique ou construction sociale? », Travaux de didactique du FLE, n55, p. 100-108.

Derivry-Plard, Martine (2008), « Comment les étudiants se représentent-t-ils leurs enseignants de langue étrangère ? », dans Pierre Martinez, Danièle Moore et Valérie Spaëth (dir.), Plurilinguismes et enseignement: identités en construction, Paris, Riveneuve, p. 141-152.

Francard, Michel, Joëlle Lambert et Françoise Berdal-Masuy (1993), L'insécurité linguistique en Communauté française de Belgique, Bruxelles, Service de la langue française de la Communauté française Wallonie-Bruxelles.

Galisson, Robert (1986), "Éloge de la didactologie/didactique des langues et des cultures (maternelle et étrangères) - (D/DLC) », Études de Linguistique Appliquée, n 64, p. 39-54.

Gauvin, Lise (2008), «Situations des littératures francophones : à propos de quelques dénominations », dans Ingse Skattum et Karin Holter (dir.), La francophonie aujourd'hui : réflexions critiques, Paris, L'Harmattan, p. 27-39. 
González, Liliana (2010), « Hacia una revolución francesa en la investigación sobre la didáctica del francés en Colombia », Signo y pensamiento, vol. 29, n57, p. 496-504.

Haugen, Einar (1962), « Schizoglossia and the Linguistic Norm », Monograph Series on Languages and Linguistics, n 15, p. 63-69.

Love, Nigel (2009), « Science, language and linguistic culture », Language \& Communication, vol. 29, $n^{\circ} 1$, p. 26-46.

Muni Toke, Valelia (2010), «La linguistique légale à la recherche du locuteur natif : de la détermination de l'origine des demandeurs d'asile», Langage et société, n 132, p. 51-75.

Muni Toke, Valelia (2013), «Le locuteur natif et son idéalisation : un demi-siècle de critiques », Histoire Épistémologie Langage, vol. 35, n², p. 5-15.

Paikeday, Thomas M. (1985), The native speaker is dead! An informal discussion of a linguistic myth with Noam Chomsky and other linguists, philosophers, psychologist, and lexicographers, Toronto/ New York, Paikeday Publishing.

Planet, Cathy (2014), « Natifs et non natifs dans l'enseignement des langues-cultures étrangères : genèse, aspects et perspectives d'un conflit de légitimités », Revista electrónica de Investigación Educativa Sonorense, $n^{\circ}$ 16, p. 86-96, disponible sur https://rediesonorense.files.wordpress. com/2014/09/revista-no-16.pdf.

Rampton, Ben (1990), «Displacing the 'native speaker': expertise, affiliation, and inheritance », ELT Journal, vol. 44, n², p. 97-101.

Rincón-Restrepo, Claudia (2016), Didactique du texte littéraire dans l'enseignement universitaire de français en Colombie : histoire, actualité et perspectives, thèse de doctorat, Paris, Université Sorbonne Nouvelle.

Rincón-Restrepo, Claudia (2020), "L'enseignement du français dans l'espace universitaire en Colombie : deux cents ans d'histoire, mais quel avenir? ", Synergies Chili, nº 16 (Du passé vers l'avenir: innovations et nouvelles compétences pour l'apprenant-citoyen du XXI siècle, sous la dir. de Marie-Noëlle Antoine et Isabel Alvarado Gutierrez), p. 49-62.

Roussi, Maria (2009), L'insécurité des professeurs de langues étrangère non natifs : le cas de professeurs grecs de français, thèse de doctorat, Paris, Université Sorbonne Nouvelle.

Silva, Haydée (2011), "Langues et territoires : le statut du français en Amérique latine », Cahier de la Chaire Senghor de la francophonie de l'Université du Québec en Outaouais, n³, disponible sur https://uqo.ca/docs/10063.

Skutnabb-Kangas, Tove et Teresa McCarty (2018), « Key concepts in bilingual education: ideological, historical, epistemological, and empirical foundations », dans Jim Cummins et Nancy Hornberger (dir.), Encyclopedia of Language and Education, vol. 5 (Bilingual Education), $3^{e}$ éd., New York, Springer, p. 3-17. [1'é éd., 1997.] 
Torres-Castillo, Claudia (2017), Culturas lingüísticas : el habitus en un mundo plurilingüe, thèse de doctorat, México, Universidad Autónoma del Estado de México.

Torres-Castillo, Claudia (2018), Enseignement du français, altérités et contacts de langues : imaginaires de professeurs mexicains, thèse de doctorat, Tours, Université de Tours.

Urbain, Jean-Didier (1982), «La langue maternelle, part maudite de la linguistique? », Langue française, n 54, p. 7-28. 